\title{
Case report: PICC line for a toddler with bilateral bidirectional Glenn shunt, Fontan circulation and persistent left superior vena
} cava

\author{
Huong Tran ${ }^{1}$, Dien Tran ${ }^{1}$, Truong Nguyen ${ }^{1}$, Nguyet Nguyen ${ }^{2}$, Mai Nguyen ${ }^{1}$, and Huyen \\ $\mathrm{NgO}^{1}$ \\ ${ }^{1}$ Vietnam National Children's Hospital \\ ${ }^{2}$ VNU Vietnam National University Hanoi
}

March 2, 2022

\begin{abstract}
Performing peripherally inserted central catheters for children with bilateral bidirectional Glenn shunt, Fontan circulation and persistent left superior vena cava differs from those with normal central venous anatomy. This study presents two PICC procedures for a toddler with this condition to demonstrate an accurate PICC approach for such children.
\end{abstract}

\section{INTRODUCTION}

Peripherally inserted central catheter (PICC) has been considered the favorable venous access protocol for children for decades. It is a light or non-sedation procedure where a catheter is inserted at a peripheral site and extended to the superior vena cava with and without the support of high-resolution ultrasound or fluoroscopic venography with contrast injected in a peripheral vein of the selected vein for PICC. ${ }^{1}$ The procedure can be implemented at the bedside or in a specialized intravenous suite by trained persons, including anesthesiologists, interventional radiologists, pediatricians or specialized IV nurses. ${ }^{1-3}$ Due to advancing into the vena cava, PICC dwelling time can be 390-575 days for infusion and blood sampling. ${ }^{1}$ The long dwelling time contributes to a decrease in venous access times; thus, reducing pain and anxiety for children. PICC-associated complications, such as thrombosis, stenosis and infection have also been reported at low levels compared to other venous access. ${ }^{1,4}$ Hence, PICC is the preferable choice for intermediate to long term venous access for medication, fluid therapy, blood sampling and parenteral nutrition.

Catheter tip location is one of the main focuses in the PICC procedure. Literature exploring PICC placement has intensively focused on descriptions of children with normal hearts and normal venous systems. ${ }^{1,2,5}$ Acceptable catheter tip locations for this group are in the superior vena cava (SVC) or inferior vena cava (IVC) depending on where the catheter is peripherally inserted, such as in upper or lower extremities. ${ }^{1,2,5}$ Acceptable catheter tip locations contribute to longer patency and lower complications such as thrombosis, phlebitis and occlusion compared to those in the non-central or left outside SVC or IVC. ${ }^{1,2,5}$ Little research has discussed the location of the catheter tip for children with abnormal heart or venous systems who might have high demands for venous access for medications or fluid therapy. This inadequate description could lead venous access teams to mistake the identification of the catheter tip location, a decrease in the success rate of the PICC procedure, and an increase in unexpected complications. This gap justifies the need to provide guidelines for PICC placement for children with congenital anomalies.

Persistent left superior vena cava (PLSVC) is a congenital anomality that should be acknowledged when performing a PICC procedure. PLSVC is a common anomaly of the thoracic venous system that is rare 
in the general population, with a prevalence of $0.3 \% .{ }^{6}$ In children with congenital heart diseases, the prevalence rate is much higher, at 4.5\%. ${ }^{7}$ PLSVC results in an unusual position of the catheter tip in the PICC procedure from a desired position in the right to the left. ${ }^{3}$

Children with single ventricular physiology and PLSVC may receive palliative surgeries, namely, a bilateral bidirectional Glenn shunt and Fontan circulation to help them survive. ${ }^{8}$ These surgical shunts also create altered central venous anatomy ${ }^{3,4}$, which could cause an unusual catheter tip location. For patient safety and procedure success, the alternative of the central venous anatomy should be reviewed and acknowledged prior to the placement of PICC.

We report two PICC procedures for a toddler having bilateral bidirectional Glenn shunt and Fontan circulation. Inadequate understanding about altered central venous anatomy in this patient prior to the PICC placement among PICC nurses resulted in some unexpected events. Experience sharing in performing a PICC for this patient aimed to identify differences in performing PICC for a child with this condition compared to children who do not and to raise awareness about the importance of reviewing venous anatomy of patients among PICC nurses prior to the procedure for the patient safety and procedure success.

\section{CASE SUMMARY}

A two year-old boy with single ventricle physiology/double outlet right ventricle/transposition of the great arteries/pulmonary stenosis, had undergone a bilateral bidirectional Glenn shunt at seven days old, in November 2019. In October 2021, he was admitted to the Heart Center, Vietnam National Children's Hospital for an elective surgery of a Fontan procedure. Three days after the Fontan procedure, the patient had chylothorax. He was then indicated to stop tube feeding and receive nutrition and medications, such as fluid therapy, lipid $20 \%$ and sandostatin $0.1 \mathrm{mg} / 1 \mathrm{ml}$ via a central venous catheter intraoperatively inserted. Nineteen days after the Fontan procedure, the patient still had chylothorax and required long-term intravenous nutritional therapy. The CVC was removed after 14 days of dwelling time, as per usual procedure of the Hospital policy. PICC then ordered to be placed for treatment purposes.

\section{7/11/2021: The first PICC procedure}

The first PICC procedure was implemented on 17 November 2021 by the PICC nurses in the Department of Cardiology in a specialized ward for procedures. Ketamine 10mg was intravenously administered to the patient prior to the procedure. Maximal sterile barriers and aseptic insertion techniques were strictly applied. The patient's veins in the right upper extremity were selected. The desired length of the catheter was $21 \mathrm{~cm}$, which was measured from the veins in the patient's right elbow to the distal to SVC. A peel-away cannula using a PICC size $24 \mathrm{G}$ from Vygon was utilized. After several attempts to access peripheral veins in the patient's elbow, a successful venous access in the cephalic vein facilitated the threading of the catheter into the vein via the peel-away cannula and advancing to the SVC. When the catheter was threaded at above $17 \mathrm{~cm}$, an unknown pressure pushed the catheter back and prevented it from advancing into the SVC. No blood return was detected. Multiple cannula attempts with the position changes of the patient's arms were made; however, the results were the same. A fifteenth cannula attempt was performed and the inserted catheter was $15 \mathrm{~cm}$ in length, with blood return. The catheter tip was verified with X-ray. The tip location was confirmed in the right axillary vein near the beginning of the right subclavian vein. The cardiologist weighed the risks and allowed the catheter to remain indwelling. A heparin dose of $2 \mathrm{U} / \mathrm{ml}$ was administered to flush the catheter. This procedure was performed without the support of high-resolution ultrasound or fluoroscopic venography with contrast. Figure 1 presents the catheter tip location of the first PICC procedure.

On 24 November 2021, the patient's skin around the right clavicle bone was red and hotter than other areas. The cardiologist and the PICC team decided to remove the catheter. Physical assessment of the patient reported no signs of PICC-associated blood infection. Blood culture showed negative. A second PICC procedure was prescribed for ongoing intravenous nutritional therapy.

\section{5/11/2021: The second PICC procedure}


The second PICC procedure was performed on 25 November 2021. The procedure was the same as the first one, but the venous access site was in the left brachial vein. The length for the catheter tip was $24 \mathrm{~cm}$, measured from the patient's left elbow to the SVC. The venous access at the peripheral left brachial vein and the cannulation step advancing to the SVC went smoothly until the inserted catheter reached $21 \mathrm{~cm}$ in length. When the catheter tip went further forward to $24 \mathrm{~cm}$ in length, flushing the catheter encountered resistance and demonstrated no blood return. The PICC nurses decided to pull the catheter back to $21 \mathrm{~cm}$ in length and asked for the verification of the catheter tip location using X-ray. The catheter tip location was confirmed in the left para mediastinal border. Figure 2 displays the catheter tip location in the second PICC.

The unexpected position of the catheter tip surprised the PICC nurses and was then explained by a cath-lab interventionist and a cardiac surgeon, who performed diagnostic catheterization and the palliative surgeries for this patient. The catheter tip was acceptably placed in the distal to the persistent left superior vena cava, only $0.5 \mathrm{~cm}$ above the left superior cavopulmonary anastomosis. Figure 3 shows the patient's PLSVC taken by diagnostic catheterization prior to the Fontan procedure on 10 November 2021.

\section{DISCUSSION}

Inadequate instructions about performing PICC for children with potential altered or abnormal central venous anatomy contributed to risks and an inaccurate approach in this patient. The PICC nurses did not have foreknowledge about the bilateral bidirectional Glenn shunt and the persistence of the left superior vena cava in this patient. This knowledge gap led to some incorrect actions when performing two PICC procedures and potential risks for this patient. Multiple attempts at cannulation in the first PICC procedure could contribute to intravascular injury and hematoma formation. For patients with a Glenn shunt, this problem could result in stenosis and thrombosis for the SVC and limit adequate passive pulmonary blood flow ${ }^{9,10}$, which may cause the obstruction of pulmonary blood flow, a life-threating event for patients with this post-operative condition. $., 9,10$ In addition, lack of awareness of the PLSVC prior to the second PICC procedure led the PICC nurses to give an inaccurate decision of the catheter tip length. The use of external pressure to thread the catheter with resistance to the target length of $24 \mathrm{~cm}$ in the SVC in the right side could contribute to injury in the left superior cavopulmonary anastomosis and facilitate hematoma formation and then thrombosis. The lack of knowledge in this topic created serious risks for this vulnerable patient. More importantly, this practice could cause multiple negative impacts for other patients with this condition in the nation-wide Heart Center in Vietnam, where about 1,000 open heart surgeries were successfully performed on a yearly basis from 2016 to 2020, in which the Glenn shunt volume was involved in about 25 to 30 cases annually.

Knowledge about PLSVC and altered central venous anatomy in children with CHD has been insufficiently discussed. While most studies and guidelines for PICC placement primarily provide instructions for children with normal hearts and central venous systems ${ }^{1,2,5}$, the acknowledgement of some altered central venous anatomy has been reviewed in only a few studies by doctors, anesthesiologists and radiologists. ${ }^{3,4}$ In Vietnam, there are no official guidelines for PICC. We note that only a few national children's hospitals can implement this procedure. Most guidelines are grey literature and largely focus on neonates with normal central venous system. Nurses perform PICC procedures with unofficial training and inadequate understanding about central venous anatomy. Therefore, it is vital to explicitly outline altered central venous anatomy in the PICC procedure for nurses to avoid preventable PICC procedure-related complications for patients. Open communication and discussion about PICC cases between doctors, surgeons and nurses about the central venous system prior to the procedure is also routinely required to avoid unexpected complications and maximize the benefits of PICC.

\section{CONCLUSION}

PICC is a favorable procedure among children with CHD for long-term medications and nutrition. Children with bilateral bidirectional Glenn shunt and/or the persistence of the left superior vena cava may have potential differences in the central venous anatomy pre- and post-operatively. These differences should be 
highlighted in PICC guidelines and reviewed by the PICC team to avoid unexpected complications for these vulnerable children.

\section{AUTHOR CONTRIBUTIONS}

TTMH: contributed to performing PICC placement, identifying research problem, synthesizing the literature and writing the manuscript. TMD and NLTT: contributed to the review of medical aspects and the central venous anatomy of the patient. NTN: involved in the research, writing and providing ideas and comments for this manuscript. NTM and NTH were the PICC nurses who performed the two PICC procedures for the patient.

\section{REFERENCES}

1. Westergaard, B., Classen, V., \& Walther-Larsen, S. (2013). Peripherally inserted central catheters in infants and children - indications, techniques, complications and clinical recommendations.Acta Anaesthesiologica Scandinavica, 57 (3), 278-287. https://doi.org/10.1111/aas.12024

2. Sharpe, E., Pettit, J., \& Ellsbury, D. L. (2013). A national survey of neonatal peripherally inserted central catheter (PICC) practices.Advances in Neonatal Care , 13 (1), 55-74. https://doi.org/10.1097/ANC.0b013e318278b907

3. Braswell, L. E. (2011). Peripherally inserted central catheter placement in infants and children. Techniques in Vascular and Interventional Radiology , 14 (4), 204-211.

4. Naik, V., Mantha, S., \& Rayani, B. (2019). Vascular access in children. Indian Journal of Anaesthesia , 63 (9), 737-745. https://doi.org/10.4103/ija.IJA_489_19

5. Mickler, P. A. (2008). Neonatal and pediatric perspectives in PICC placement. Journal of Infusion Nursing , 31 (5), 282-285. https://doi.org/10.1097/01.NAN.0000336182.77656.4e

6. Sacchetti, A. (1998). The science and practice of pediatric cardiology. Pediatric Emergency Care, 14 (4), 310. https://doi.org/10.1097/00006565-199808000-00020

7. Perles, Z. M. D., Nir, A. M. D., Gavri, S. M. D., Golender, J. M. D., TaShma, A. M. D., Ergaz, Z. M. D., \& Rein, A. J. J. T. M. D. (2013). Prevalence of persistent superior vena cava and association with congenital heart anomalies. The American Journal of Cardiology ,112 (8), 1214-1218. https://doi.org/10.1016/j.amjcard.2013.05.079

8. Rao, P. S. (2015). Fontan Operation: Indications, short and long term outcomes. Indian Journal of Pediatrics , 82 (12), 1147-1156. https://doi.org/10.1007/s12098-015-1803-6

9. Kaipa, S., Mastropietro, C. W., Bhai, H., Lutfi, R., Friedman, M. L., \& Yabrodi, M. (2020). Upper body peripherally inserted central catheter in pediatric single ventricle patients. World Journal of Cardiology , 12 (10), 484-491. https://doi.org/10.4330/WJC.V12.I10.484

10. Miller, J. W., Vu, D. N., Chai, P. J., Kreutzer, J. H., John, J. B., Vener, D. F., \& Jacobs, J. P. (2013). Upper body central venous catheters in pediatric cardiac surgery. Pediatric Anesthesia ,23 (11), 980-988. https://doi.org/10.1111/pan.12261 


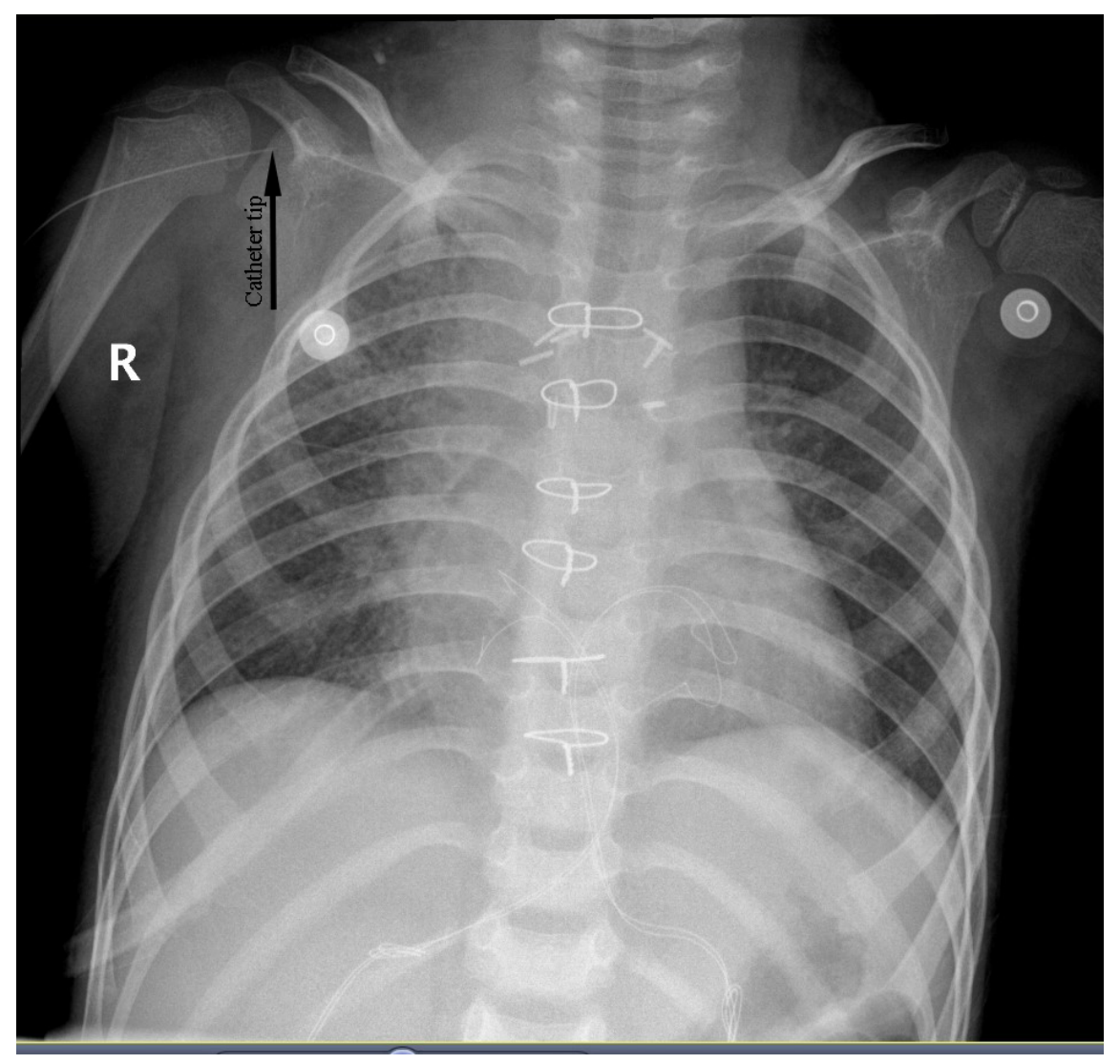




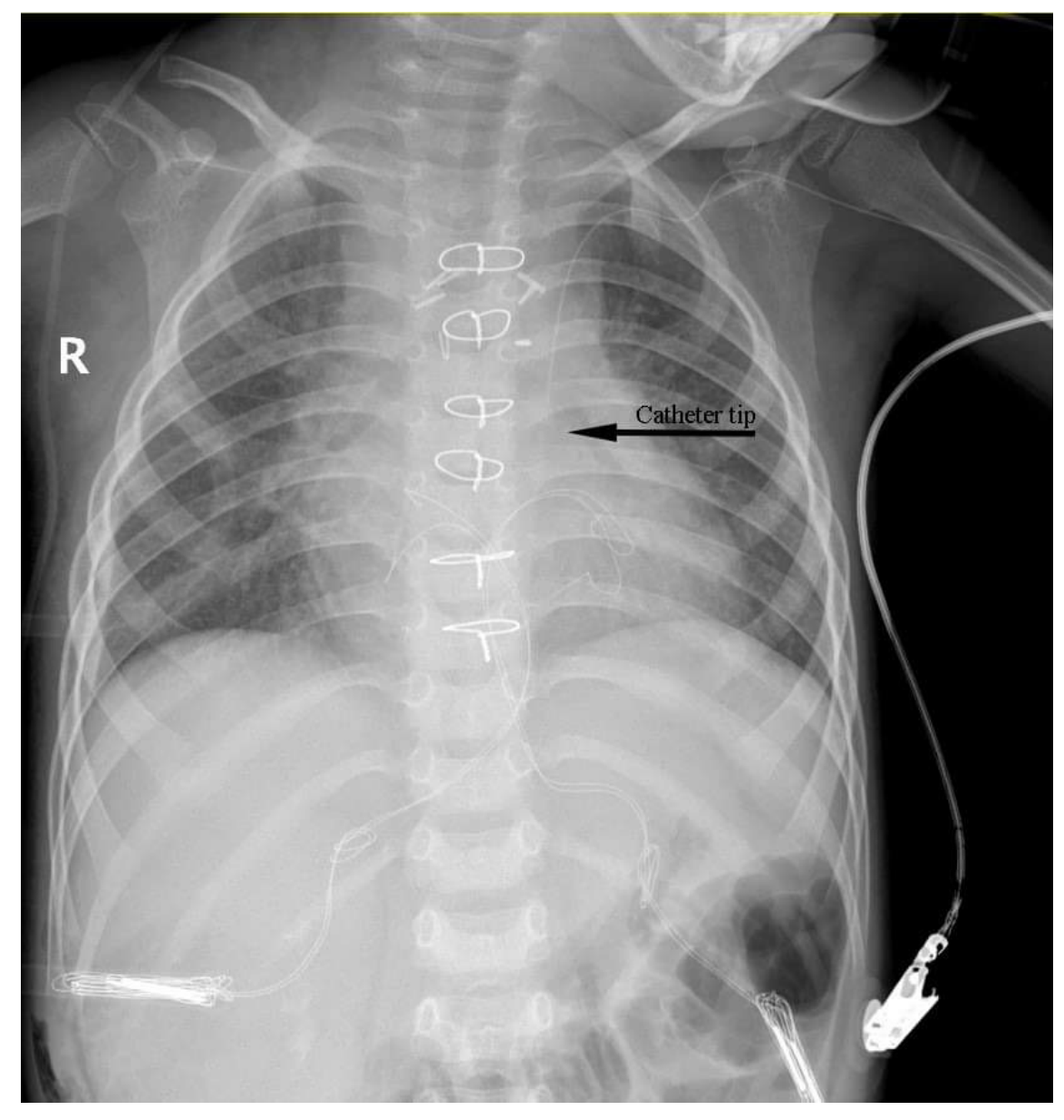




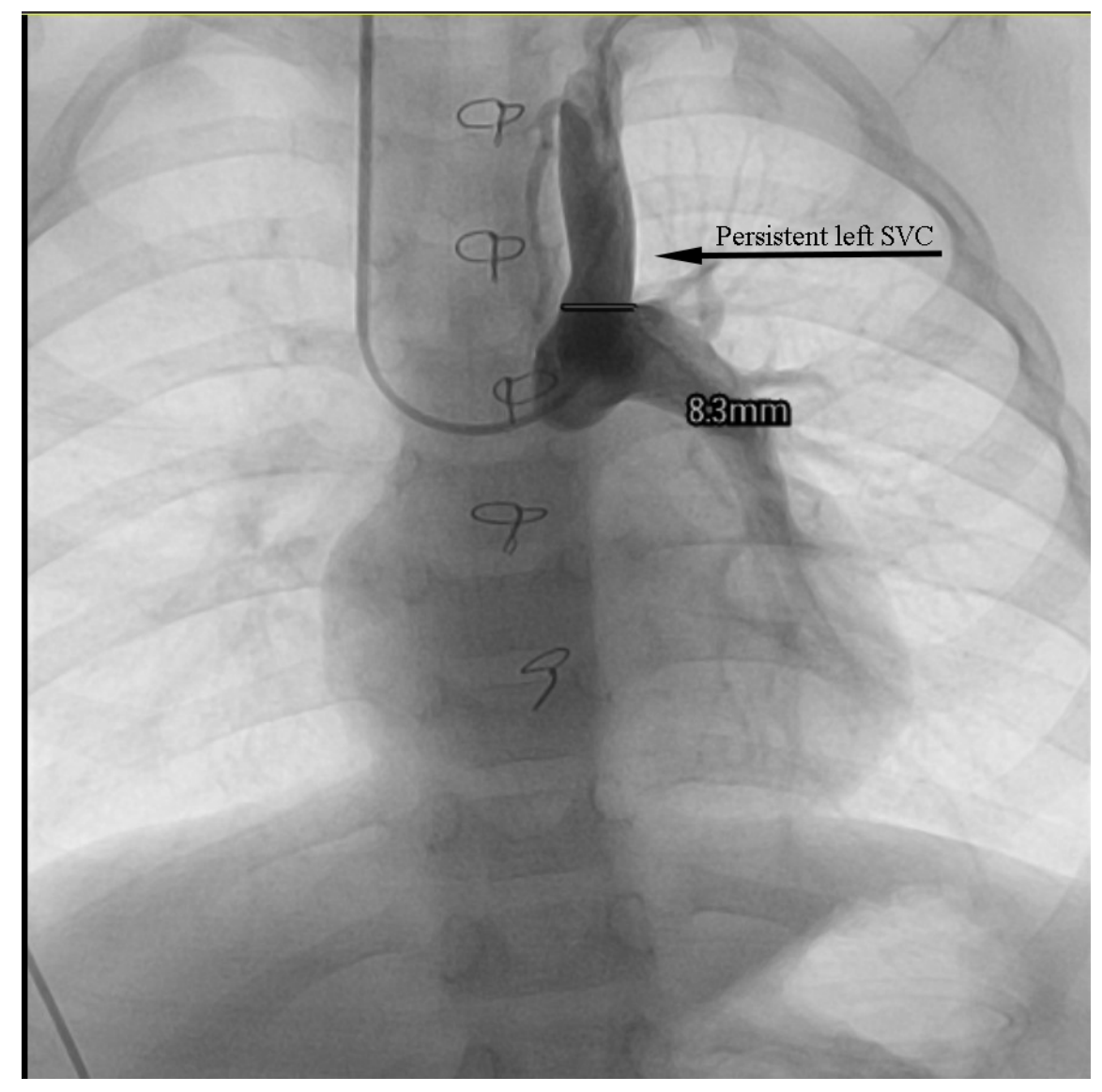

